\title{
Comparison of the morphology of vital and dead human spermatozoa
}

\author{
J. P. P. Tyler and N. G. Crockett* \\ Department of Obstetrics and Gynaecology, Westmead Centre, Westmead, New South Wales 2145 \\ and ${ }^{*}$ Department of Statistics, Macquarie University, North Ryde, New South Wales 2113, \\ Australia
}

\begin{abstract}
Summary. The distribution of different morphological types of spermatozoa have been compared in the live and dead populations of an eosin-stained dry film. There were significant differences between the distribution of sperm types within each of the 80 individuals studied $(P<0.05)$ and between the two groups taken as a whole $(P<$ 0.001 ). Normal oval, and small oval forms appeared preferentially in the vital population while spermatozoa with defects of the tail were preferentially found in the dead population. It is concluded that assessment of the total number of functional spermatozoa in an ejaculate should be made by reference to the percentage of normal oval forms in the live population.
\end{abstract}

\section{Introduction}

Gross abnormalities of human sperm morphology are rare, or are sometimes a transient phenomenon. Generally, $>60 \%$ of spermatozoa in the ejaculate are morphologically normal, excluding this characteristic as a major cause of reduced fertility in men (Eliasson, 1977a). However, in the overall assessment of a semen sample, diagnosis is made by reference to the total number of morphologically normal, motile spermatozoa per ejaculate. Since motility is necessary for successful fertilization it would seem reasonable that the morphology of living spermatozoa would correlate better with potential fertility than would the overall morphology of the ejaculate, particularly if the percentage of dead spermatozoa was high. Staining methods for differentiating between live and dead spermatozoa have long been available but no further experiences with this simple technique have been published since Fredricsson, Waxegard, Brege \& Lundberg's (1977) call for more research.

The purpose of this study was to compare the distribution of different morphological types of spermatozoa in the live and dead populations and to relate these findings to the overall number of dead spermatozoa in the ejaculate.

\section{Materials and Methods}

The data for this study were obtained from 80 semen samples delivered for infertility investigations. Supravital staining of spermatozoa was carried out within $1 \mathrm{~h}$ of ejaculation by examining a dry smear from $100 \mu \mathrm{l}$ of well-mixed semen and $200 \mu \mathrm{l}$ of stain $(0.67 \%$ eosin Y, $5 \%$ nigrosin in distilled water). This concentration of eosin has been shown to be most suitable for staining dead spermatozoa (Eliasson, 1977b). Each morphological quantitation (\% dead, live and 
dead distribution) was undertaken by examining 200 spermatozoa appearing consecutively under the microscope with $\times \mathbf{4 0 0}$ magnification and bright-field or Nomarski optics. First, the frequency of dead spermatozoa was determined and then the morphology of spermatozoa in the live and dead populations. The morphological evaluation was based on the recommendations of WHO (1980) and only mature spermatozoa possessing a head, mid-piece and tail were scored. So-called "pinheads" (motile tails) were excluded, as were spermatozoa trapped in aggregates where their form was unclear. Immature cells could not be quantitated because they could not be distinguished from other round cells with the vital stain. The 7 morphological types distinguished were large, small and normal oval types, tapered or cigar-shaped forms (in which the head width was greatly diminished in relation to head length), duplicate forms having multiple heads or tails (this category took precedence over other classifications), tail defects (spermatozoa having severely coiled tails or tails that were broken or bent at right angles to the mid-piece) and amorphous forms (spermatozoa with bizarre structural defects of the head which could not be placed into any of the other categories). The frequency of spermatozoa with a cytoplasmic droplet was so low in the series ( 9 found in 32000 spermatozoa) that this classification was omitted from the statistical analysis.

\section{Results and Statistical analysis}

In a preliminary experiment, the distribution of dead spermatozoa across the smear was examined. For 19 samples, 10 groups of 100 spermatozoa at different sites on the smear were examined to determine the percentage of dead spermatozoa. Variation could be large when only 100 spermatozoa were analysed but for groups of 200 the distribution was more stable, the coefficient of variation being up to 3 times greater for groups of 100 than for groups of 200. In samples that exhibited extreme variability, any extremely large values were significantly different from their mean at the $5 \%$ level for groups of 100 but not for groups of 200 . There was no advantage in counting more than 200 spermatozoa. In a second experiment defining the system, the number of spermatozoa necessary to quantitate morphology was examined by analysing 1000 consecutive spermatozoa from 11 specimens. Similar methods indicated that the stability of different morphological types was adequate once 200 spermatozoa had been counted.

The overall difference between the live and dead morphological types is shown in Text-fig. 1. The normal oval and small spermatozoa were principally found in the live population, whereas

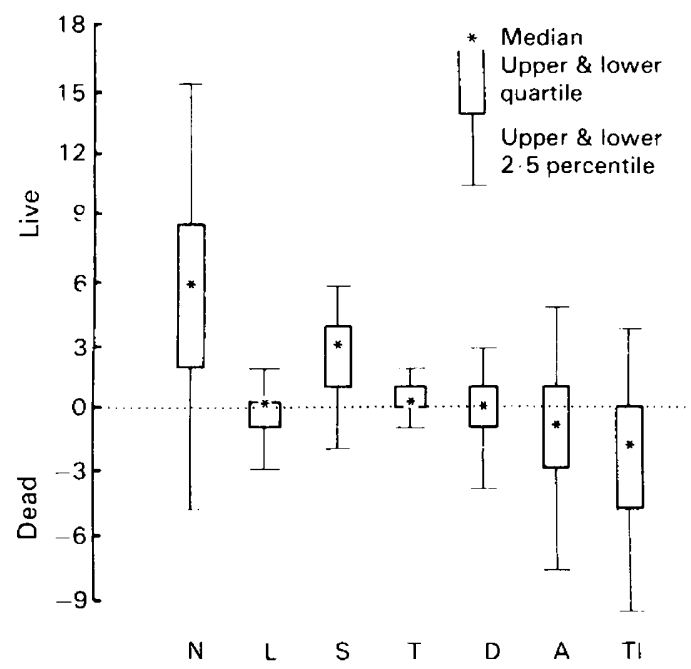

Text-fig. 1. The differences between the morphology of live and dead human spermatozoa. $\mathrm{N}$ $=$ normal oval forms $; \mathbf{L}=$ large-headed forms $; \mathrm{S}=$ small-headed forms $; \mathrm{T}=$ tapered forms; $\mathrm{D}=$ duplicates $\mathbf{A}=$ amorphous forms and $\mathrm{TI}=$ tail defects. 
spermatozoa with defects of the tail, and to a lesser extent amorphous types, were generally found in the dead population.

When a comparison of all 7 categories of sperm morphology for the live and dead populations was made within each individual, over the whole sample of 80 subjects, a $\chi^{2}$ test of fit for multinomials yielded a value of 1096, with 539 degrees of freedom; there are therefore significant differences in the distribution of morphological types in the live and dead population $(P<0 \cdot 001)$. When the live and dead morphology was compared for each individual, 40 of the 80 subjects exhibited differences at the $5 \%$ level of significance and 24 of these 40 reached the $1 \%$ level.

Similarly, when only the percentage of normal oval spermatozoa was compared for all 80 subjects between the live and dead populations, the $\chi^{2}$ analysis gave a value of $265 \cdot 5$, showing significant differences between the groups $(P<0.001)$. The mean percentage difference between the live and dead population was $4.4 \%$, the median being $5.3 \%$. Again, if each individual was considered, 24 subjects exhibited significant differences between the live and dead groups at the $5 \%$ significance level.

\section{Discussion}

An assessment of sperm morphology is a pre-requisite of semen analysis, allowing evaluation of the status of testicular germ cell production and providing indicators to factors contributing to infertility. For instance, excessive numbers of tapered and immature forms can be indicative of viral infection and a high percentage of coiled tails may define prostatic dysfunction, but the correlation of varicocoele with a high incidence of tapered forms has recently been questioned (Rodriguez-Rigau, Smith \& Steinberger, 1981).

However, evaluation of sperm morphology remains subjective, and interpretation of standard definitions can differ between technicians and laboratories (Fredricsson, 1978). Since all the analyses in this study were carried out by one person the contribution to variance from this source was minimal. Similarly, there are a number of staining procedures available, such as the classic techniques (i.e. Papanicolaou), a method recommended by WHO (1980), fluorescence (Tejada, Mavik, Winer \& Friedman, 1981) or the novel pre-stained slides (Testsimplets) (Schoenfeld, Amelar, Dubin \& Amelar, 1981), but each of these have their disadvantages and all may be suspect since the report of Mortimer (1981) who ascribed functional differences to spermatozoa when viewed by light microscopy or by surface replica electron microscopy. While Mortimer (1981) did not suggest the involved electron microscopic technique should be used for routine seminology his final comment was "assessment of sperm morphology from vitally-stained preparations may represent a useful innovation".

Since there is little one can recommend if gross abnormalities of sperm morphology are consistently apparent, it would seem logical, as proposed by Fredricsson et al. (1977), to relate fertility potential to the motility of the vital population. This has often been used by animal physiologists but has seen little application by clinical seminologists. The examination of a dry eosin : nigrosin-stained film is easy and economical in cost and time but can lead to an overestimate of the number of dead spermatozoa present (Burgos \& di Paolo, 1951). However, simultaneous estimation of morphology and vitality using the multiple exposure technique of Makler (1979) still presents many practical problems for most routine laboratories and is unlikely to be the method of choice for some time. The results of this study confirm and extend those of Fredricsson et al. (1977) and show that significant differences occur in the distribution of morphological types between the live and dead populations, and that this is independent of the percentage of dead spermatozoa present. The techniques used however differ in that bright field and Nomarski optics were used rather than phase-contrast microscopy which can make visualization of eosin-stained spermatozoa difficult. Furthermore, in our much larger series ( 80 rather than 22 ) the analyses were made within individuals rather than across all individuals in a group. To remove the effects of large differences 
between individuals comparisons were made of all sperm types within each individual simultaneously and then aggregated over the total sample. Thus each individual acted as his own control and the effects of large differences between individuals were removed.

The finding that normal and small oval forms are preferentially distributed in the live population while spermatozoa with tail defects (excluding duplicates) preferentially appeared as non-vital is novel. Normal oval forms and small oval spermatozoa consistently show good progressive motility in ejaculates and it is probable that these forms should therefore be correlated best with fertility. Similarly, spermatozoa recovered from the pouch of Douglas at operation show a consistently better overall morphology (Mortimer, Leslie, Kelly \& Templeton, 1982) although to date there have been few reports in the literature. Mortimer (1978), in reviewing the literature for possible selection of spermatozoa in the female reproductive tract, concluded that spermatozoa select themselves for transport and that quality of motility is one of the most important characteristics. The finding that tapered forms appear with a higher frequency, and that amorphous forms appear with a lower frequency, in the live population confirms the results of Fredricsson et al. (1977), but in contrast, spermatozoa with tail defects were not equally distributed and were preferentially seen amongst dead spermatozoa. This finding confirms the observations of Mortimer et al. (1982) that such spermatozoa are less likely to traverse the cervix.

A mean difference of $4.4 \%$ (albeit statistically significant) between the percentage of normal oval forms in the live and dead populations may not appear startling from a practical view point, but the range of differences spans $28 \%$, showing marked variation between individuals. It is concluded, therefore, that assessment of the total number of functional spermatozoa in an ejaculate should be made by reference to the percentage of normal oval spermatozoa found in the live population.

\section{References}

Burgos, M.H. \& di Paolo, G. (1951) Eosin test for the evaluation of sperm vitality. Fert. Steril. 2, 542-544.

Eliasson, R. (1977a) Semen analysis and laboratory workup. In Male Infertility; Workup, Treatment \& Research, pp. 169-188. Eds A. T. K. Cockett \& R. L. Urry. Grune \& Stratton, London.

Eliasson, R. (1977b) Supravital staining of human spermatozoa. Fert. Steril. 28, 1257.

Fredricsson, B. (1978) Morphologic evaluation of spermatozoa in different laboratories. Andrologia 11, 57-61.

Fredricsson, B., Waxegard, G., Brege, S. \& Lundberg, I. (1977) On the morphology of live spermatozoa of human semen. Fert. Steril. 28, 168-174.

Makler, A. (1979) Simultaneous differentiation between motile, non-motile, live and dead human spermatozoa combining supravital staining and multiple exposure photography procedures. Int. J. Androl. 2, $32-42$.

Mortimer, D. (1978) Selectivity of sperm transport in the female genital tract. In Spermatozoa, Antibodies and Infertility, pp. 37-53. Eds J. Cohen \& W. F. Hendry. Blackwell, Oxford.

Mortimer, D. (1981) The assessment of human sperm morphology in surface replica preparations for transmission electron microscopy. Gamete Res. 4, 113-119.

Mortimer, D., Leslie, E.E., Kelly, R.W. \& Templeton, A.A. (1982) Morphological selection of human spermatozoa in vivo and in vitro. J. Reprod. Fert. 64, 391-399.

Rodriguez-Rigau, L.J., Smith, K.D. \& Steinberger, E. (1981) Varicocele and the morphology of spermatozoa. Fert. Steril. 35, 54-57.

Schoenfeld, C., Amelar, R.D., Dubin, L. \& Amelar, S. (1981) A new staining technique for the rapid determination of the morphologic characteristics of sperm. Fert. Steril. 36, 408-410.

Tejada, R.I., Mavik, J.J., Winer, J.H. \& Friedman, S. (1981) A new metachromatic fluorescent stain for rapid morphologic evaluation of human sperm. Fert. Steril. 36, 535-537.

WHO (1980) Laboratory manual for the examination of human semen and semen-cervical mucus interaction, p. 16. Press Concern. Singapore.

Received 29 March 1982 